\title{
ASYMPTOTIC TRACTS OF HARMONIC FUNCTIONS III
}

\author{
KARL F. BARTH \\ Department of Mathematics \\ Syracuse University \\ Syracuse, NY 13244, USA \\ e-mail: kfbarth@mailbox.syr.edu
}

DAVID A. BRANNAN

The Open University Department of Pure Mathematics

Milton Keynes MK7 6AA, United Kingdom

e-mail: D.A.Brannan@open.ac.uk

(Received June 30, 1995)

\begin{abstract}
A tract (or asymptotic tract) of a real function $u$ harmonic and nonconstant in the complex plane $\mathcal{C}$ is one of the $n_{c}$ components of the set $\{z: u(z) \neq c\}$, and the order of a tract is the number of non-homotopic curves from any given point to $\infty$ in the tract. The authors prove that if $u(z)$ is an entire harmonic polynomial of degree $n$, if the critical points of any of its analytic completions $f$ lie on the level sets $\tau_{\jmath}=\left\{z: u(z)=c_{\jmath}\right\}$, where $1 \leq \jmath \leq p$ and $p \leq n-1$, and if the total order of all the critical points of $f$ on $\tau_{\jmath}$ is denoted by $\sigma_{\jmath}$, then

$$
\left\{n_{c}: c \in \Re\right\}=\{n+1\} \cup\left\{n+1+\sigma_{\jmath}: 1 \leq \jmath \leq p\right\} .
$$
\end{abstract}

KEY WORDS AND PHRASES: Asymptotic tracts, harmonic functions.

1991 SUBJECT CLASSIFICATION CODES: Primary: 31A05; Secondary: 30C35.

\section{INTRODUCTION}

This paper continues a study, begun in [1] and [2], of the asymptotic tracts of functions harmonic in $\mathcal{C}$ (entire harmonic functions).

Definition 1. An asymptotıc tract (or tract) of a real function $u(z)$ harmonic and nonconstant in $\mathcal{C}$ is a component of the set $\{z: u(z) \neq c\}$ for some real number $c$.

It was shown in [1] that each tract $T$ is necessarily simply-connected and unbounded, and that $u$ is necessarily unbounded in each tract $T$; in addition, $\infty$ is an accessible boundary point (in $\mathcal{C}$ ) of each tract $T$. The local mapping properties of analytic functions show that the set $\{z: u(z) \neq c\}$ consists of a finite or countable number of curves which are locally analytic, except at the zeros of $f^{\prime}(z)$ (where $f^{\prime}$ is any analytic completion of $u$ )-where the set $\{z: u(z)=c\}$ branches. Observe that the angle between the 'branches' must be equal to $2 \pi / n$ for some $n \geq 1$.

We continue the study of harmonic polynomials in the plane initiated in [3], where it was shown that, if $u(z)$ is a harmonic polynomial in $\mathcal{C}$ of degree $n$, then the number, $k$, of tracts of $u$ satisfies the sharp inequality

$$
n+1 \leq k \leq 2 n
$$

A special case of our results, putting Example 2 together with Theorem 1, shows that, given any pair of positive integers $n$ and $k$ that satisfy the inequality (1) there is a harmonic polynomial $u(z)$ of degree $\mathrm{n}$ with $k$ tracts. This is stronger than [3, Theorem 3] where it was shown that there exists a harmonic polynomial of degree $n$ that has $2 n$ tracts for the case $c=0$. We also discover a restriction, for each given harmonic polynomial $u(z)$ in $\mathcal{C}$, on the number of tracts of $u(z)-c$, as the constant $c$ varies over $\Re$.

Definition 2. An unbounded simply-connected domain $T$ in $C$ is sald to be branched of order $n_{T}$ (possibly $n_{T}=+\infty$ ) if it has the following property: There exists a family $\mathcal{T}_{T}$ of $n_{T}$ nonhomotopıc ( $\imath n T$ ) and disjoint (except for the end-point $z_{T}$ ) Jordan curves in $T$ connecting some fixed point in $T, z_{T}$ say, to $\infty$; in additıon, any Jordan curve in $T$ joinıng $z_{T}$ to $\infty$ is homotopic 
(in $T$ ) to one of the elements of $\mathcal{T}_{T}$. If $n_{T}=1$, we say that $T$ is unbranched; if $n_{T}<+\infty$, we say that $T$ is finitely branched; if $n_{T}=+\infty$, we say that $T$ is infinitely-branched.

\section{NUMBERS OF TRACTS}

Let $u(z)$ be an entıre harmonic polynomial of degree $n$. Then, if $z=r e^{\imath \theta}$, we have that

$$
u(z)=a_{n} r^{n} \cos \left(n \theta+\theta_{n}\right)+O\left(r^{n-1}\right), \text { where } a_{n} \neq 0 .
$$

It follows that near $\infty$ there must be on $\{z \cdot|z|=r\}$ at least $n$ arcs (each of angular length about $\pi / n$ ) on which $u(z)>0$, and at least $n$ arcs (each of angular length about $\pi / n$ ) on which $u(z)<0$. Since $u$ is a polynomial of degree $n$ and so can have at most $2 n$ zeros on $\{z:|z|=r\}$, it follows that for sufficiently large $r$ there are precisely $n$ arcs of each type. Also, it is easy to prove that the boundaries separating the $2 n$ regions comprising $\{z:|z|=r, u(z) \neq 0\}$ tend to radial lines of angular separation $\pi / n$ as $r \rightarrow+\infty$.

We will denote by $n_{c}$ the number of components of the set $\{z: u(z)-c \neq 0\}$. It will be useful to examine how $n_{c}$ varies with $c$. For sufficiently large $r$, the set $\{z:|z|>R\} \cap\{z: u(z) \neq 0\}$ consists of precisely $2 n$ unbounded disjoint domains. Then, for such an $r$, we define

$$
M=1+\max \{u(z):|z| \leq r\} .
$$

It follows that the set $\{z: u(z)-M \neq 0\}$ has exactly $n$ components in which $\{z: u(z)-M>0\}$ and exactly one component in which $\{z: u(z)-M<0\}$. Thus $n_{M}=n+1$. Also, it follows from the Phragmen-Lindelörf Principle that $n_{c}=n+1$ when $c>M$. We now look at how $n_{c}$ varies as $c$ decreases from the value $M$. The components (tracts) of $\{z: u(z)-c \neq 0\}$ vary continuously with $c$, in terms of kernel convergence. Hence, as $c$ decreases, $n_{c}$ is an integer and varies continuously with $\mathrm{c}$ (hence remains constant) - except at those values of $c$ for which a critical point of the analytic completion of $u$ lies on the set $\{z: u(z)=c\}$.

Now two tracts of $u(z)-c$ in which $u(z)-c$ has opposite signs can never lie in a single tract of $u(z)-c_{1}$, for $c_{1} \neq c$, since $u$ is unbounded in any tract; however their boundaries may meet in a point or in an arc. No two tracts of $u(z)-c$ can have the property that their boundaries meet in a set with more than one component: for, if they did, then there would be a bounded (non-empty) domain on whose boundary $u(z)=c$, and so we would have $u(z) \equiv c$ in $\mathcal{C}$.

Suppose that $T_{1}$ and $T_{2}$ are two tracts of $u(z)-c$ in which $u(z)-c>0$; we will call such tracts upper tracts (for the value $c$ ). (Lower tracts are defined similarly.) It may be that $\partial T_{1} \cap \partial T_{2}=\emptyset$. However we cannot have a situation where $\partial T_{1} \cap \partial T_{2}$ contains an arc in $\mathcal{C}$, by the Maximum Principle. It follows, then, that, if $\partial T_{1}$ meets $\partial T_{2}$, the set $\partial T_{1} \cap \partial T_{2}$ must be a singleton.

If $T_{1}$ and $T_{2}$ are both upper tracts or both lower tracts for which $\partial T_{1} \cap \partial T_{2}=\left\{z_{0}\right\}$, then there must exist an equal number of upper and lower tracts whose boundaries contain $z_{0}$. Since $z_{0}$ must thus be a critical point of any analytic completion of $u$, there can be at most $(n-1)$ such points $z_{0}$ (since $u$ is a polynomial of degree $n$ ). Note also that, as $c$ decreases, the upper tracts individually increase in size. Hence their total number must decrease as $c$ decreases.

Our main result in this Section is the following.

Theorem 1. Let $u(z)$ be an entire harmonıc polynomial of degree $n$. Let the critıcal points of any of its analytıc completıons $f$ lue on the level sets $\tau_{\jmath}=\left\{z: u(z)=c_{\jmath}\right\}$, where $1 \leq \jmath \leq p$ and $p \leq n-1$, and let the total order of all the critıcal points of $f$ on $\tau_{j}$ be denoted by $\sigma_{j}$. (In partıcular, $\sum_{\jmath=1}^{p} \sigma_{\jmath}=n-1$.) Then $\left\{n_{c}: c \in \Re\right\}=\{n+1\} \cup\left\{n+1+\sigma_{\jmath}: 1 \leq \jmath \leq p\right\}$.

Proof. Let $f$ be any analytic completion of $u$.

Case 1. All the critical points of $\mathrm{f}$ lie on different level sets for $\mathrm{u}$.

Assume first that all the critical points of $f$ are simple; then we may choose our notation so that they lie on the level sets $\tau_{\jmath}=\left\{z: u(z)=c_{\jmath}\right\}, 1 \leq \jmath \leq n-1$, where $c_{1}>c_{2}>\ldots>c_{n-1}$. Then, by the previous comments, for $c>c_{1}$ (for example, when $c=M$ (see (3)), we have $n_{c}=n+1$ and there are $n$ upper tracts of $u$ and one lower tract. Next, $n_{c_{1}}=n+2$ and there are, for the value $c=c_{1}, n$ upper tracts and two lower tracts (the lower tract has 'split' in two). Finally, for $c_{1}>c>c_{2}$, we have $n_{c}=n+1$, and there are $(n-1)$ upper tracts (two upper tracts have 'combined') and 2 lower tracts.

As $c$ decreases further, a similar argument holds for each $c_{\jmath}$ in turn, $2 \leq \jmath \leq n-1$. For $c_{\jmath-1}>c>c_{\jmath}$, we have $n_{c}=n+1$ and there are $(n+1-\jmath)$ upper tracts and $\jmath$ lower tracts; when $c=c_{\jmath}$, we have $n_{c_{\jmath}}=n+2$ and there are $(n+1-\jmath)$ upper tracts and $(\jmath+1)$ lower tracts; and, for $c_{\jmath}>c>c_{\jmath+1}$ (with the convention that $c_{n}=-\infty$ ), we have $n_{c}=n+1$ and there are $(n-\jmath$ ) upper tracts and $(\jmath+1)$ lower tracts. 
Assume next that the critical points of $f$ are not necessarily simple. First, suppose that the level set $\left\{z: u(z)=c_{\jmath}\right\}$, for some particular value of $\jmath$, contains a critical point of $f$ (at $z_{\jmath}$ where $f^{\prime}$ has a zero of order $b_{\jmath}$ ). Let $I$ be an open interval of $\Re$ that contains $c_{\text {j }}$ but contains no other $c$ 's corresponding to critical points of $f$ Then, for a sufficiently small neighborhood $\mathcal{U}$ of $z$, there are $\left(2 b_{\jmath}+2\right)$ tracts of $u(z)-c_{\jmath}$ that meet $\mathcal{U}$, namely $\left(b_{\jmath}+1\right)$ upper tracts and $\left(b_{\jmath}+1\right)$ lower tracts. However, when $c>c_{\jmath}, c \in I$ and $c-c_{\jmath}$ is sufficiently small, there are only $\left(b_{\jmath}+2\right)$ tracts of $u(z)-c$ that meet $\mathcal{U}$, namely $\left(b_{j}+1\right)$ upper tracts and 1 lower tract; similarly, when $c<c_{\jmath}, c \in I$ and $c_{\jmath}-c$ is sufficiently small, there are $\left(b_{\jmath}+2\right)$ tracts of $u(z)-c$ that meet $\mathcal{U}$, namely $(b,+1)$ lower tracts and 1 upper tract.

Now consider the level set $\{z: u(z)=c\}$ for an arbitrary $c$. Since, except for values of $c$ corresponding to critical points of $f$ (and even then locally only in small neighborhoods of the critical points themselves) the tracts vary continuously with $c$ (in the sense of kernel convergence), it follows from the above argument that there is some number $N$ such that, for $\left|c-c_{\jmath}\right|$ sufficiently small and non-zero, we have $n_{c}=N+1$ whereas $n_{c_{3}}=N+1+b_{\jmath}$. But $n_{M}=n+1$, so that we must have $N=n$. This completes the proof of Case 1 of the theorem.

Case 2. More than one critical point of $f$ lies on a given level set for $u$.

Assume first that, for some $c_{\jmath}$, the level set $\left\{z: u(z)=c_{\jmath}\right\}$ contains just two branch points, $z_{1}$ and $z_{2}$, of orders $b_{1}$ and $b_{2}$ respectively, and that $z_{1}$ and $z_{2}$ lie on different components, $C_{1}$ and $C_{2}$ respectively, of $\left\{z: u(z)=c_{\jmath}\right\}$; thus $C_{1} \cap C_{2}=\emptyset$. It follows that there exists some Jordan curve from $\infty$ to $\infty$ that separates $C_{1}$ from $C_{2}$; this curve can be chosen to lie either in a single component of $\left\{z: u(z)>c_{\jmath}\right\}$ or in a single component of $\left\{z: u(z)<c_{\jmath}\right\}$. By considering the local behavior of $u$ near $z_{1}$ and $z_{2}$, and by using the fact that components of $\{z: u(z)-d \neq 0\}$ vary continuously with $d$ (except when their boundaries coalesce), it follows that, when $\left|d-c_{\jmath}\right|$ is sufficiently small, we have $n_{d}=n+1$ and $n_{c_{j}}=(n+1)+b_{1}+b_{2}$. A similar argument works in the case of more than two branch points on a single level set of $u$, so long as each such branch point lies on a different component of that level set.

Assume next that, for some $c_{\jmath}$, the level set $\left\{z: u(z)=c_{\jmath}\right\}$ contains just two branch points, $z_{1}$ and $z_{2}$, of orders $b_{1}$ and $b_{2}$ respectively (corresponding to zeros of $f^{\prime}$ of these orders), and that $z_{1}$ and $z_{2}$ lie on the same component, $C$, of $\left\{z: u(z)=c_{\jmath}\right\}$. Then there is a Jordan subarc $\Gamma$ of $C$ joining $z_{1}$ to $z_{2}$; let $z^{\prime}$ be any interior point of this subarc. Since $C$ cannot contain any closed Jordan curves, it follows that there are precisely two tracts, $T_{1}$ and $T_{2}$, say, of $u(z)-c_{\jmath}$ that have $\Gamma-\left\{z_{1}, z_{2}\right\}$ as part of their boundaries; we may assume that $u(z)>c_{\jmath}$ in $T_{1}$ and so that $u(z)<c_{j}$ in $T_{2}$. Similar considerations also show that there is a Jordan curve $J_{1}$ in $T_{1} \cup\left\{z^{\prime}\right\}$ that joins $z^{\prime}$ to $\infty$ inside $T_{1}$, and a Jordan curve $J_{2}$ in $T_{2} \cup\left\{z^{\prime}\right\}$ that joins $z^{\prime}$ to $\infty$ inside $T_{2}$.

We define $J^{\prime}=J_{1} \cup J_{2}$. Then $J^{\prime}$ plays the same role as $J$ did earlier (when it separated $C_{1}$ from $C_{2}$ ), and a similar argument to the previous one shows that

$$
n_{d}= \begin{cases}n+1, & \text { if } d \neq c_{\jmath}, \\ n+1+\left(b_{1}+b_{2}\right), & \text { if } d=c_{\jmath}\end{cases}
$$

Again a similar argument can be used even when there are more than two branch points on the same component of the level set.

The result of this theorem is stronger than [3, Theorem 1], where it was shown that $\left\{n_{c}: c \in \Re\right\}$ is a subset of $\{n+1, n+2, \ldots, 2 n\}$.

Notice that for the function $u_{1}(z)=\operatorname{Re}\left(z^{n}\right)$ we have $n_{0}=2 n$ and $n_{1}=n+1$, and that in fact $\left\{n_{c}: c \in \Re\right\}=\{n+1,2 n\}$. The next two examples show that, while this particular function $u_{1}$ is extremal in a certain sense, the conclusion of Theorem 1 concerning the range of possible values of $n_{c}$ (as $c$ varies) is best-possible.

Example 1. There exists a harmonıc polynomıal $u$ of degree $n$ for whıch $\left\{n_{c}: c \in \Re\right\}=$ $\{n+1, n+2\}$, and all the critıcal points of any analytıc completion $f$ of $u$ are simple and lie on different level sets of $u$.

Let $u(z)=\operatorname{Re}\left(z^{n}-A z\right)$, for a complex number $A$ yet to be specified. The analytic completion $f(z) \equiv z^{n}-A z$ of $u$ has critical points where $n z^{n-1}-A=0$; that is, where

$$
z=z_{k}=\left(\frac{A}{n}\right)^{\frac{1}{n-1}} \exp \left(\frac{2 \pi \imath k}{n-1}\right), \quad 1 \leq k \leq n-1
$$


Now $u\left(z_{k}\right)=\operatorname{Re}\left(\frac{z_{k} A(1-n)}{n}\right)$; and it follows that, if $A$ is chosen with $|A|=1$ and with $\arg A$ not a rational multiple of $2 \pi$, then all the values of $\left\{u\left(z_{k}\right)\right\}_{1}^{n-1}$ are distinct. Thus $u$ has the desired properties.

Example 2. Let $p$ be an integer, such that $1 \leq p \leq n-1$, and let $b_{1}, b_{2}, . ., b_{p}$ be any integers in $[1, n-2]$ for which $\sum_{j=1}^{p} b_{\jmath}=n-1$. There exists a harmonıc polynomıal u $(z)$ of degree $n$ with the properties that any analytıc completion $f$ of $u$ has critıcal points of orders $b_{1}, b_{2}, \quad,, b_{p}$, and that all these critical points lie on different level sets of $u$. Hence (from Theorem 1)

$$
\left\{n_{c}: c \in \Re\right\}=\{n+1\} \cup\left\{n+1+b_{\jmath}: 1 \leq \jmath \leq p\right\} .
$$

First, let $f_{1}$ be the polynomial given by $f_{1}(0)=0$ and

$$
f_{1}^{\prime}(z)=(z-1)^{b_{1}}\left(z-a_{2}\right)^{b_{2}} z^{(n-1)-\left(b_{1}+b_{2}\right)}
$$

where $a_{2}$ is chosen to be either $\frac{1}{2}$ or to be very close to $\frac{1}{2}$; in particular, we make our choice of $a_{2}$ to ensure that $u_{1}(1) \neq 0$, where $u_{1}(z) \equiv \operatorname{Re} f_{1}(z)$. It follows from Rolle's Theorem that the points 1 and $a_{2}$ lie on different level sets of $u_{1}$.

Next, let $f_{2}$ be the polynomial given by $f_{2}(0)=0$ and

$$
f_{2}^{\prime}(z)=(z-1)^{b_{1}}\left(z-a_{2}\right)^{b_{2}}\left(z-a_{3}\right)^{b_{3}} z^{(n-1)-\left(b_{1}+b_{2}+b_{3}\right)},
$$

where $a_{3}$ is positive but small. By continuity arguments (on $f$ ) we see that we may choose $a_{3}$ sufficiently near to 0 that 1 and $a_{2}$ lie on different level sets of $u_{2}(z) \equiv \operatorname{Re} f_{2}(z)$.

We have to check that $a_{3}$ can be chosen so that $a_{3}$ lies on a different level set of $u_{2}$ from those that contain either 1 or $a_{2}$. But, if $a_{3}$ is sufficiently small, we have that

$$
\begin{aligned}
u_{2}(1) & \approx \int_{0}^{1}(t-1)^{b_{1}}\left(t-a_{2}\right)^{b_{2}} t^{(n-1)-\left(b_{1}+b_{2}\right)} d t, \\
u_{2}\left(a_{2}\right) & \approx \int_{0}^{a_{2}}(t-1)^{b_{1}}\left(t-a_{2}\right)^{b_{2}} t^{(n-1)-\left(b_{1}+b_{2}\right)} d t,
\end{aligned}
$$

and

$$
u_{2}\left(a_{3}\right) \approx(-1)^{b_{1}+b_{2}+b_{3}}\left(a_{2}\right)^{b_{2}}\left(a_{3}\right)^{\left(n-\left(b_{1}+b_{2}\right)\right)} \int_{0}^{1}(1-x)^{b_{3}} x^{(n-1)-\left(b_{1}+b_{2}+b_{3}\right)} d x
$$

hence, for all sufficiently small $a_{3}$, the values of $u_{2}(1), u_{2}\left(a_{2}\right)$ and $u_{2}\left(a_{3}\right)$ are all distinct.

A similar argument shows, after a further $(p-3)$ steps, that the polynomial $u(z) \equiv \operatorname{Re} f(z)$, where $f(0)=0$ and

$$
f^{\prime}(z)=(z-1)^{b_{1}}\left(z-a_{2}\right)^{b_{2}}\left(z-a_{3}\right)^{b_{3}}\left(z-a_{4}\right)^{b_{4}} \ldots\left(z-a_{p}\right)^{b_{p}}
$$

and the sequence $\left\{a_{3}\right\}_{j=3}^{p}$ decreases to 0 sufficiently quickly, has the desired properties.

Finally, as was mentioned in the Introduction, suppose $n$ and $k$ are positive integers such that $n+1 \leq k \leq 2 n$. If $k=n+1$, we see from Example 1 that there exists a harmonic polynomial $u(z)$ such that $n_{c}=\{n+1, n+2\}$. If $n+1<k \leq 2 n$ and we set $b_{1}=k-(n+1)$ and $b_{2}=2 n-k$, Example 2 shows that there exists a harmonic polynomial $u(z)$ such that

$$
\left\{n_{c}\right\}=\left\{n+1, n+1+b_{1}, n+1+b_{2}\right\}=\{n+1, k, 3 n+1-k\} .
$$

ACKNOWLEDGMENT. The first author gratefully acknowledges support from the Science and Engineering Research Council of the United Kingdom.

\section{REFERENCES}

1. K. F. Barth and D. A. Brannan, Asymptotıc tracts of harmonıc functıons I, Acad. Sci. Fenn. Series A. I. Math. 11 (1986), 215-232.

2. $35-52$.

3. W. K. Hayman D. A. Brannan, W. H. J. Fuchs and Ü. Kuran, A characterısatıon of harmonıc polynomials in the plane, Proc. London Math. Soc. (3) 32 (1976), 213-229. 


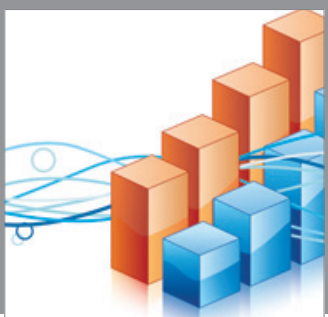

Advances in

Operations Research

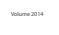

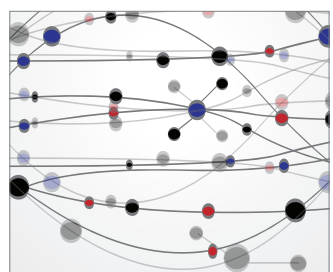

\section{The Scientific} World Journal
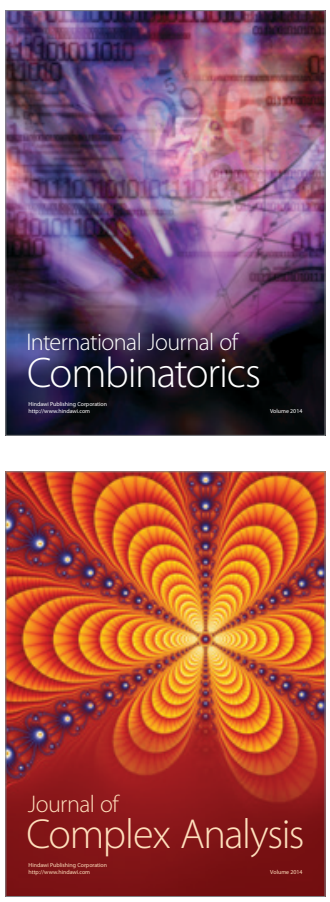

International Journal of

Mathematics and

Mathematical

Sciences
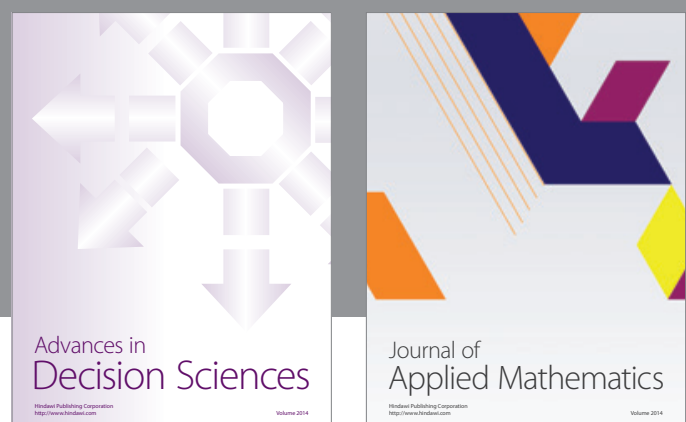

Journal of

Applied Mathematics
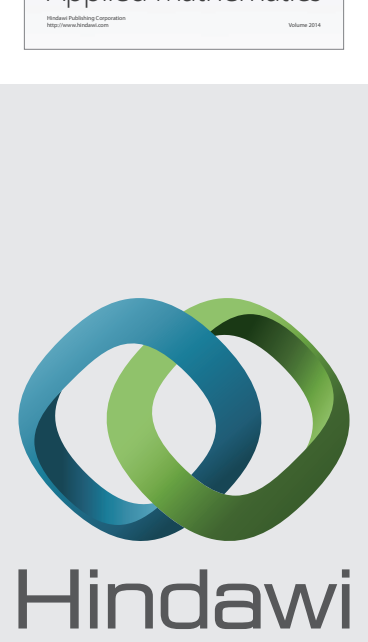

Submit your manuscripts at http://www.hindawi.com
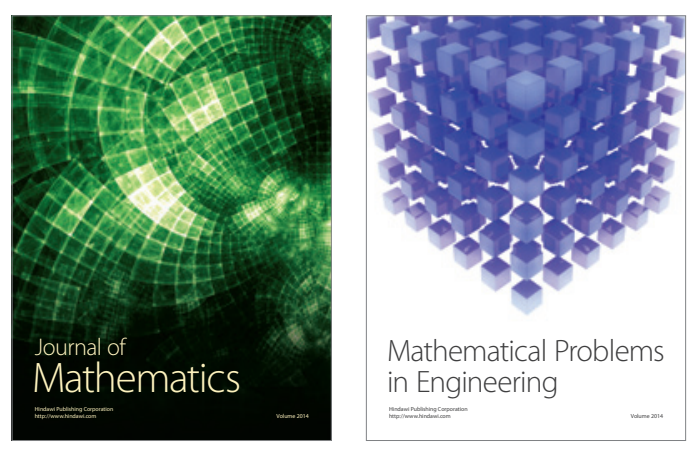

Mathematical Problems in Engineering
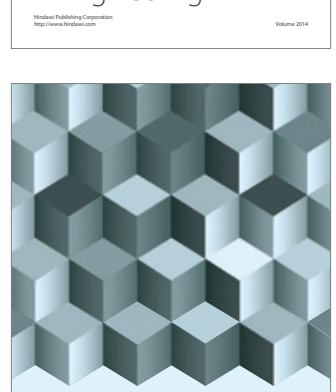

Journal of

Function Spaces
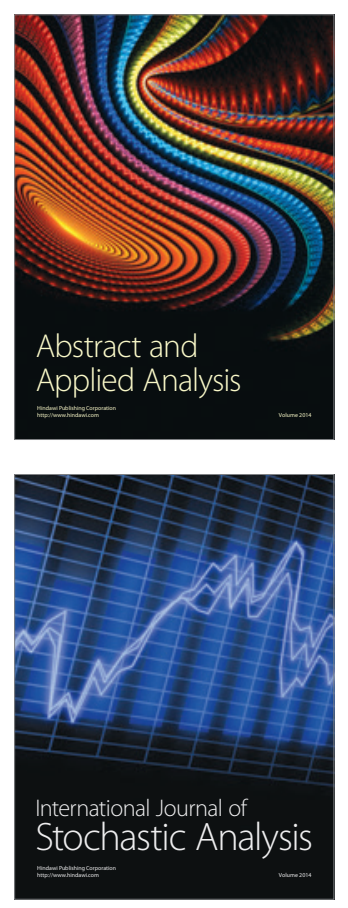

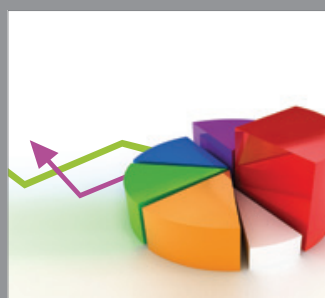

ournal of

Probability and Statistics

Promensencen
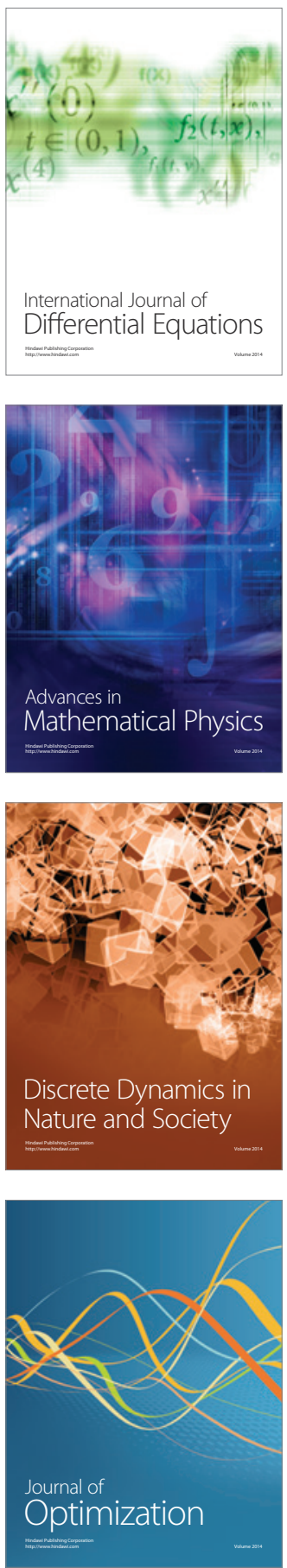